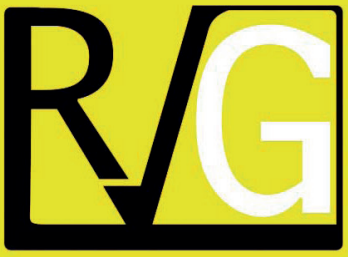

Julio - Septiembre, 2021

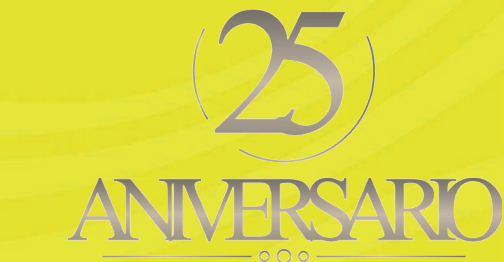

ANMERSARO
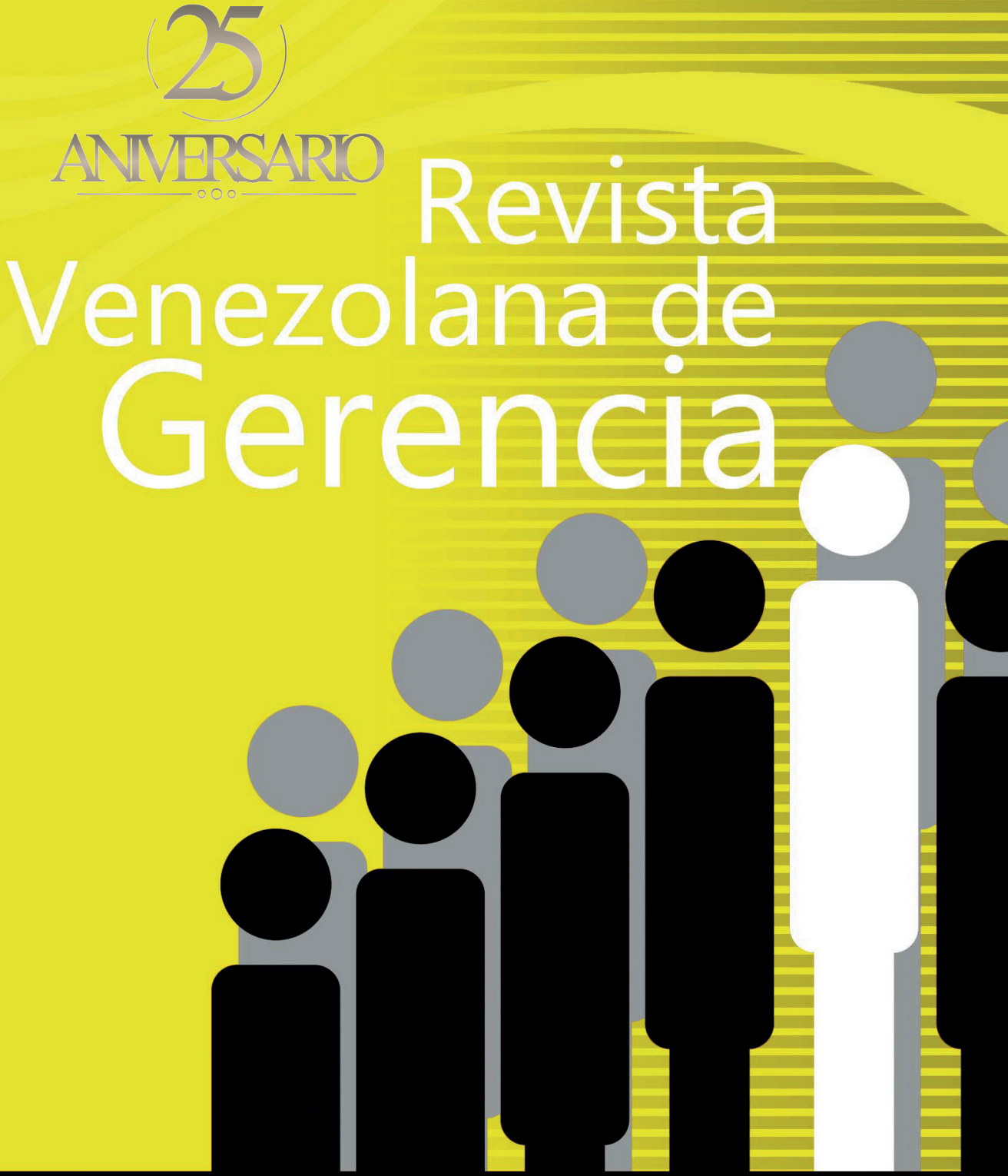

UNIVERSIDAD DEL ZULIA (LUZ)

Facultad de Ciencias Económicas y Sociales Centro de Estudios de la Empresa

ISSN 1315-99

Esta obra está bajo una licencia de Creative Comm Reconocimiento-NoComercial-Compartirlgual 3.0 Unpo http://creativecommons.org/licenses/by-nc-sa/3.0/deed.es 
COMO CITAR: Matadamas, I. A., Cruz, B. C., y Toledo, A. (2021). Economía solidaria en redes familiares de artesanos en San Pedro Cajonos. Revista Venezolana de Gerencia, 26(95), 514-529. https://doi. org/10.52080/rvgluz.27.95.5
Universidad del Zulia (LUZ)

Revista Venezolana de Gerencia (RVG)

Año 26 No. 95 Julio-Septiembre 2021, 514-529

ISSN 1315-9984 / e-ISSN 2477-9423

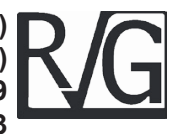

\title{
Economía solidaria en redes familiares de artesanos en San Pedro Cajonos
}

\author{
Matadamas Cruz, Irlanda Abigail* \\ Cruz Cabrera, Blasa Celerina** \\ Toledo López, Arcelia***
}

\section{Resumen}

En la actualidad, la economía solidaria se ha caracterizado por ser un modelo alternativo al existente, y su base son los principios de cooperación, igualdad, empleo, para una transformación social, sin embargo esta economía no es adquirida en cualquier tipo de estructuras de trabajo. En algunas comunidades del Estado de Oaxaca es muy común trabajar con los principios de una economía solidaria. La presente investigación tiene como objetivo describir un contexto de economía solidaria en redes familiares de artesanos de la comunidad de San Pedro Cajonos, Villa Alta, Oaxaca. Ya que, en las actividades artesanales las estructuras de trabajo se basan en la cooperación y solidaridad de los integrantes de esas redes. La investigación se realiza con la metodología cualitativa, con las visitas de campo a la comunidad e identificando las redes familiares de artesanos se observó como se manifiesta un contexto de economía solidaria, identificando la cooperación y cohesión social en los procesos productivos de las actividades artesanales: artesanías de madera y textiles de hilo de seda, generando en el seno de la comunidad un desarrollo económico local.

Palabras clave: Economía solidaria; artesanos; redes familiares.

Recibido: 05.03 .21

Aceptado: 10.05 .21

* Estudiante del Doctorado en Ciencias en Desarrollo Regional y Tecnológico, Tecnológico Nacional de México/Instituto Tecnológico de Oaxaca, México, irlanda.mc86@gmail.com (autor para correspondencia) Orcid: https://orcid.org/0000-0002-0534-1950

** Profesor investigador del Departamento de División de Estudios de Posgrado e Investigación, Tecnológico Nacional de México/Instituto Tecnológico de Oaxaca, México, cabreracruz85@hotmail.com. Orcid: https:// orcid.org/0000-0003-4694-4261

*** Profesor investigador del Departamento de Administración de Recursos Naturales, Instituto Politécnico Nacional/CIIDIR Oaxaca, México, artoledol@ipn.mx. Orcid: https://orcid.org/0000-0002-2328-5438 


\title{
Solidarity economy in family networks of artisans in San Pedro Cajonos
}

\begin{abstract}
At present, the solidarity economy has been characterized as an alternative model to the existing one, and its basis is the principles of cooperation, equality, employment, for a social transformation, however this economy is not acquired in any type of work structure. In some communities in the State of Oaxaca it is very common to work with the principles of a solidarity economy. The present research aims to describe a context of solidarity economy in family networks of artisans in the community of San Pedro Cajonos, Villa Alta, Oaxaca. Since, in artisanal activities, work structures are based on the cooperation and solidarity of the members of these networks. The research is carried out with qualitative methodology, with field visits to the community and identifying the family networks of artisans, it was observed how a context of solidarity economy manifests itself, identifying cooperation and social cohesion in the productive processes of artisan activities: wooden handicrafts and silk thread textiles, generating local economic development within the community.
\end{abstract}

Keywords: Solidarity economy; artisans; family networks.

\section{Introducción}

El sector solidario existe a nivel mundial con sus principios que no son los mismos que los que aplican en el sector privado, sino que tienen un objetivo firme que es la búsqueda del bien común y la solidaridad, además de mejorar la calidad de vida de una comunidad, en conjunto con un enfoque de desarrollo (social, cultural, ambiental).

Como lo señalan Razeto (1989:50) y Fajardo (2008:50), la economía solidaria es una formulación teórica de nivel científico, diseñada a partir de experiencias prácticas que, más allá de sus diferencias, comparten diversos rasgos constitutivos esenciales de solidaridad, mutualismo, cooperación y autogestión, compartidos por los trabajadores o la comunidad.
Como se menciona anteriormente, los rasgos de solidaridad y cooperación se identifican en la comunidad, y principalmente en su actividad económica, ya que, las actividades que producen para subsistir, es fundamental mencionar que son de carácter social, como menciona Orellana (2007:3), ya que, son realizadas por una comunidad: las relaciones económicas, como cualquier tipo de relación establecida entre personas, son relaciones sociales.

Bajo ese contexto de economía solidaria, en la comunidad de San Pedro Cajonos, Villa Alta, Oaxaca existen dos actividades artesanales: artesanías de madera y artesanías textiles de hilo de seda, ambas actividades las desarrollan redes familiares de artesanos, y presentan la siguiente problemática: durante el trabajo 
artesanal se transmite el conocimiento de generación en generación, es decir, con una dinámica transgeneracional, esto es, el conocimiento ancestral y la identidad cultural; junto con la estructura productiva, como la forma de organizarse y la participación familiar en el proceso artesanal y el valor social (cooperación y solidaridad) que los artesanos crean en la comunidad y las artesanías. Es por ello, que se tiene como objetivo describir un contexto de economía solidaria en redes familiares de artesanos de la comunidad de San Pedro Cajonos, Villa Alta, Oaxaca, mediante la metodología cualitativa descriptiva con una observación y aplicación de una entrevista no estructurada a 10 representantes de las redes familiares identificadas.

\section{Revisión Teórica sobre economía solidaria}

La economía solidaria (también conocida como economía social tuvo su origen en el siglo XVIII, con el impulso del cooperativismo. La segunda revolución industrial fortaleció al capitalismo y, con ello, la polarización social. En este contexto surgió el cooperativismo como respuesta a la convulsión económica y social imperante, sin embargo, la economía solidaria quedó marginada como modo de producción al fortalecerse al hacerse dominante el capitalismo (Orellana, 2007:7).

A partir de los años 80 , dentro del nuevo contexto de globalización neoliberal, frente el fracaso del sistema capitalista de responder a las verdaderas necesidades materiales, mentales y espirituales de la humanidad, y al fracaso de los caminos alternativos que se intentaron desarrollar (el socialismo por ejemplo), surgieron iniciativas desde la propia sociedad civil que buscaron ser dar respuesta real a los problemas generados por la globalización capitalista $y$, a la vez, ser iniciativas o alternativas transformadoras profundas.

De acuerdo con lo mencionado anteriormente y considerando a la economía solidaria como un modo de producción al igual que la economía tradicional, cuando se exponen las características y los elementos "ideales" de la economía solidaria, se asume que, evidentemente, en la realidad, existen matices y situaciones intermedias que no, por ello, dejan de tener valor como ejemplos de formas alternativas de organización y de hacer economía.

Así mismo, Marcos Arruda, en el IV Forum Social Mundial -Panel "Por una economía del pueblo: realidades y estrategias de lo local y a lo global", Mumbai India (2004)definióa la economía solidaria como "una forma ética, reciproca y cooperativa de consumir, producir, intercambiar, financiar, comunicar, educar, desarrollarse que promueve un nuevo modo de pensar $y$ de vivir" $y$, además, asevera este modelo económico pretende tener como premisas (Calderon, 2008:51): a) La sociedad civil, especialmente el mundo del trabajo, empoderase para ser el sujeto principal y soberano de su vida y de su propio desarrollo. El Estado, el capital, el desarrollo económico y el tecnológico son concebidos como medios para hacer viable el desarrollo humano y social;

b) La colaboración solidaria es la forma predominante de relación social;

c) El compartir y la coparticipación en la producción, distribución y consumo son la emoción y la matriz de la acción;

d) El Estado democrático es un proyecto común, cuyo papel es de promover un sistema de sociabilidad 
basado en la cooperación, el respeto mutuo y el pleno desarrollo de cada ciudadano y comunidades;

e) La democracia es realizada como la construcción de un sistema humano y social, un espacio socioeconómico, mental y psíquico del compartir, del respeto mutuo, de la cooperación y de la coparticipación; y

f) La matriz cultural es que quienes trabajan -el individuo social- son los legítimos líderes y, por tanto, comparten el poder y la responsabilidad por la toma de las decisiones. En una palabra, es una cultura del individualismo social o del personalismo colectivo o, más aun, del socialismo individual.

Dentro de este marco, él término economía social y solidaria puede tener dos significados principales complementarios (Coraggio, 2008) en donde es evidente que solidaridad es inseparable de corresponsabilidad social como lo menciona Puig et al. (2016:25). Es un sistema económico cuyo funcionamiento asegura la base material integrada con una sociedad justa y equilibrada o es un proyecto de acción colectiva (incluyendo prácticas estratégicas de transformación y cotidianas de reproducción dirigido a contrarrestar las tendencias socialmente negativas del sistema existente con la perspectiva -actual o potencialde construir un sistema económico alternativo que responda al principio ético ya enunciado (Coraggio, 2013:4).

Por ello es importante definir términos que son comunes de escuchar social y solidario: el primero se utiliza para reafirmar la negatividad que provoca el mercado libre, que tiende a mercantilizar todas las actividades de la vida social y como consecuencia hay destrucción de los vínculos sociales y solidarios. Implica defender la cohesión social, no necesariamente $\mathrm{y}$, probablemente, no de las sociedades que han resultado del reinado neoliberal, sino de las que pueden resultar de la acción voluntaria de la construcción de otra sociedad éticamente construida (Puig et al. 2016:26).

La segunda palabra es "solidario" y que no sólo se base en la rivalidad y la competencia de todos contra todos, en el no reconocimiento del otro y sus necesidades, en la irresponsabilidad por los efectos de las acciones estratégicas (desde la producción hasta el consumo) sobre la sociedad y su naturaleza (Puig et al. 2016:26).

Todo lo que existe, en sí, son problemas sociales, pobrezas y exclusiones masivas, todo ello por el mismo capitalismo global. Por el contrario, la solidaridad y la reciprocidad son condiciones positivas para la transición y constitutivas de una economía diferente, que sume al contexto de manera integral, en lugar de restar o generar impactos negativos al entorno.

\subsection{Principios de la economía solidaria}

Se denomina economía solidaria al sistema socioeconómico, cultural y ambiental desarrollado de forma individual o colectiva a través de prácticas solidarias, participativas, humanistas y sin ánimo de lucro para el desarrollo integral del ser humano como fin de la economía (Celorio \& López de Munain, 2007:110).

Se reconocen, en este espacio, todas aquellas iniciativas asociativas, empresariales, económicas y financieras que trabajen bajo los principios siguientes según los autores Celorio \& López de Munain (2007): 
1. Igualdad. Promover la igualdad en las relaciones y satisfacer, de manera equilibrada, los intereses de todas las personas protagonistas en las actividades de la empresa o de la organización.

2. Empleo. Crear empleo estable, favoreciendo, especialmente, el acceso de personas en situación o riesgo de exclusión social, asegurando a cada persona condiciones de trabajo y una remuneración digna, estimulando su desarrollo personal y la asunción de responsabilidades.

3. Medio ambiente. Favorecer acciones, productos y métodos de producción respetuosos con el medio ambiente.

4.Cooperación. Favorecer la cooperación en lugar de la competencia, dentro y fuera de la organización.

5. Sin carácter lucrativo. Las iniciativas solidarias tienen como fin principal la promoción humana y social, por lo que son de carácter esencialmente no lucrativas. Los beneficios revertirán a la sociedad mediante el apoyo a proyectos sociales, a nuevas iniciativas solidarias o a programas de cooperación al desarrollo, entre otros.

6. Compromiso con el entorno. Las iniciativas solidarias estarán comprometidas con el entorno social en el que se desarrollan, lo que exige la cooperación con otras organizaciones, así como la participación en redes, como camino para que experiencias solidarias concretas puedan generar un modelo socioeconómico alternativo (pág. 108).

Con estos principios de economía solidaria se explica cuyo concepto se basa en las dinámicas de la sociedad civil y en la solidaridad (Klein et al. 2014:7) y se ofrece como marco para abordar el cambio social "desde abajo" o bien desde la base de la pirámide.
Las relaciones sociales susceptibles de propiciar una transformación de niveles (Hillenkamp \& Bessis, 2012:85), no únicamente en el ámbito social, es decir, también desde un enfoque integral.

\subsection{Dimensiones de la economía solidaria}

Coraggio (2013:4) plantea que, para fomentar el buen vivir se necesitan cuatro equilibrios: el equilibrio de los seres humanos consigo mismo; el equilibrio entre los seres humanos; el equilibrio de los seres humanos con la naturaleza y el equilibrio entre las comunidades de seres humanos. Lo que propone Coraggio sobrepone la satisfacción de las necesidades de las personas antes que la acumulación del capital. Es contrario a la visión marcada por la acción individual y del mercado autorregulado que está violentando a los seres humanos y la naturaleza, es una irracionalidad de lo irracional y que está llevado a la humanidad al filo del precipicio.

La economía solidaria incorpora la propuesta de la economía ecológica, al considerar que los sistemas económicos no son cerrados en los flujos monetarios y reales, sino que son sistemas abiertos que están relacionados con la biosfera, en donde los procesos de producción, distribución, consumo y postconsumo generan flujos energéticos entrópicos $\mathrm{y}$, por tanto, se promueven formas alternativas de producir, intercambiar y consumir que consideren el desgaste energético que produce la actividad económica (Yaselca \& Jara, 2013) citado en Puig et al. (2016:114).

Con los elementos señalados anteriormente, se debe mencionar que la economía solidaria no sólo tiene que mirarse desde la clasificación 
económica, sino que aborda varias dimensiones, las cuales permiten tener una mirada integral de ella (Yaselca \& Jara, 2013).

Estas cinco dimensiones del diagrama 1 son inclusivas y, además, es muy importante mencionar que es integral ya que incluye aspectos que una economía de mercado no puede incluir por su propia naturaleza.

\section{Diagrama 1 \\ Dimensiones de la economía solidaria}

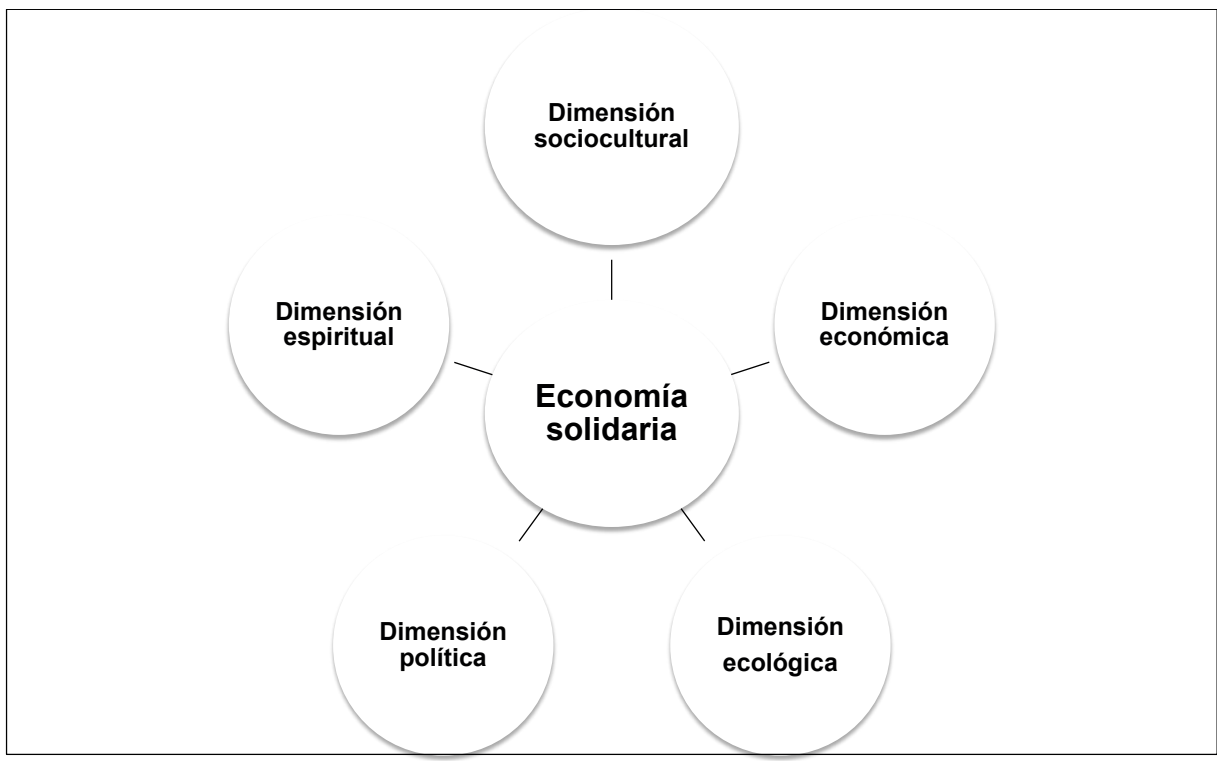

Fuente: Yaselca \& Jara (2013).

La dimensión espiritual, en la comunidad donde se desarrolla la investigación, se da en el momento en que los artesanos y la comunidad, en sí, se relaciona con la naturaleza mediante la generación de vínculos, ya que, con lo que ella proporciona, la comunidad y, de manera natural, los artesanos pueden elaborar sus artesanías que es una de las principales fuentes de materia prima (árboles de aguacatillo y el árbol de morera) principalmente.
Por otra parte, en la comunidad donde se desarrolla la investigación, está la dimensión cultural donde se promueven las relaciones económicas, valorizándolas como prácticas económicas y también como componentes de la cultura que han perdurado de forma ancestral como lo es el caso del patrimonio cultural que consiste en danzas que caracterizan a los habitantes de la comunidad mencionada y que usan máscaras de 
madera elaboradas por ellos mismos.

La relación que hay entre la naturaleza y los habitantes de la comunidad se establece en los procesos económicos mediante relaciones amigables con el medio ambiente en donde la producción, la distribución, el consumo y el postconsumo se pueden determinar ya que los artesanos hacen uso más adecuado de los recursos naturales con que cuentan y, a esto, se le llama dimensión ecológica.

Una referencia de cómo son las relaciones dentro de la comunidad se puede describir mediante la dimensión política, contexto institucional y de poder de usos y costumbres que se dan en la comunidad donde se desarrollará la investigación, y que es aquí donde se generan normas sociales para cuidar los recursos de la comunidad.

\section{Aproximaciones metodológicas del estudio}

Las visitas de trabajo de campo en la comunidad San Pedro Cajonos, Villa Alta, Oaxaca, comunidad donde se recolecta actualmente información, mediante observación a las actividades que realizan las redes familiares de artesanos.

El espacio donde se localiza la comunidad en la cual desarrollará la presente investigación de acuerdo a los datos descritos en el plan de desarrollo municipal (2010) de San Pedro Cajonos, indica que se localiza al noreste de la capital del Estado de Oaxaca.

Sus límites, determinados en la Resolución Presidencial del año 2008 , señalan que sus linderos son los siguientes, al Norte: Colinda con los terrenos comunales de la comunidad de San Francisco Cajonos, Zoochila y Santa Catarina Yahuio, sirviendo como lindero desde el punto trino "Rio Trapiche" o "Hamaca" hasta llegar a otra mojonera que se llama "Leg-Biche" o "Rio Grande".

Al Noreste limita con el pueblo de Santiago Laxopa hasta llegar a la mojonera donde nace un arroyo. En los límites del pueblo de San Miguel del Valle colinda hasta llegar al paraje denominado "Gran Bedaguio". Al Sur colinda con Santo Domingo Xagacia y tiene otra colindancia con el rio de Xagacia que es límite natural. Al Este tiene a San Francisco Cajonos y el punto trino es el río Cajonos. Al Oeste colinda con la comunidad de San Miguel Cajonos, San Miguel del Valle y con la comunidad de Santa María Yavesia.

Por la distribución de los pueblos vecinos, San Francisco y San Miguel como se muestra en la ilustración 1 , existen dos versiones sobre el nombre de Cajonos, una es porque la comunidad participo en la muerte de los fiscales santos proporcionando una tabla para la hechura de sus cajones para el entierro, la otra es porque estos pueblos están entre cerros como encajonados, y es así como es adaptado el nombre "Cajonos" por los habitantes de la comunidad. 


\section{Ilustración 1 Mapa del municipio de San Pedro Cajonos, Villa Alta}

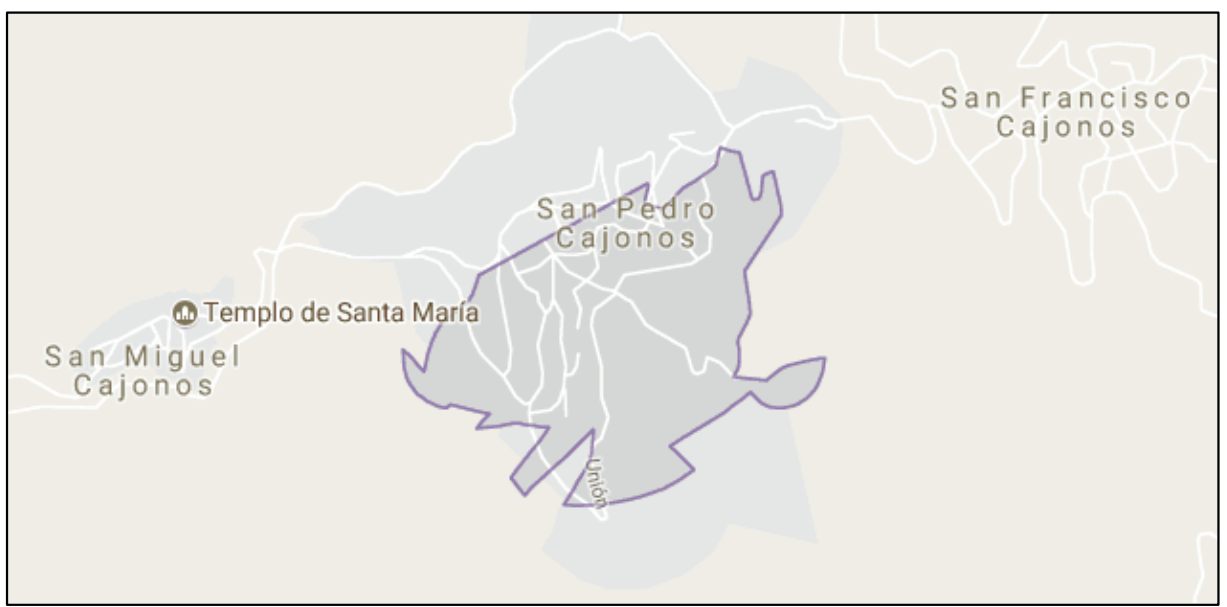

Fuente: INEGI. Marco Geo estadístico Municipal 2015, INEGI. Información Topográfica.

Su distancia aproximada a la capital del Estado es de 90 kilómetros, aproximadamente a dos horas cuarenta minutos, a diferencia de otras comunidades que poseen una gama de artesanías su cercanía a la ciudad es de quince a veinte minutos, lo que complica a los artesanos de madera y seda el acceso para comercializar y promocionar sus productos.

En la comunidad existen sistemas de producción como tal, es en el sector secundario, en industria y transformación, el tallado de madera, y la crianza del gusano de seda y de esto se desprende la producción de artesanías de seda.
La forma en la que se organizan los artesanos de madera es por familias, los trabajos los realizan en sus hogares y se involucran todos los integrantes de familia, desde lo mas jovenes o niños que se encargan de recolectar las ramas secas en el bosque, otros ya comienzan con el tallado de la madera dando forma según la rama o tronco de árbol puede ser una pequeña ave como el colibri que es característico en la comunidad o un jaguar a tamaño real, y finalmente la decoración de la figura ya terminada con la técnica proporcionada por los maestros artesanos de más edad como se muestra a continuación en el diagrama 2. 


\section{Diagrama 2 \\ Elaboración de artesanías de madera}

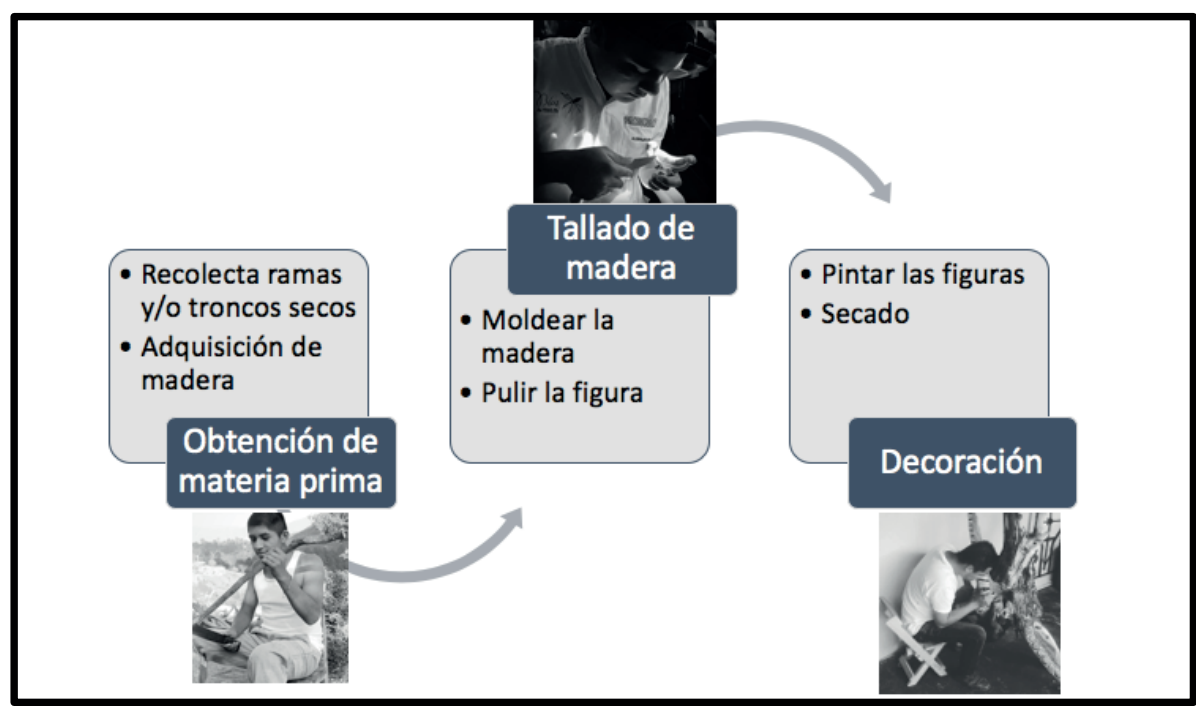

Fuente: elaboración propia basada en la Información observada en investigación de campo 2019.

La inversión de tiempo y cuidados para la crianza del gusano de seda y producción de artesanías de seda es muy alto, ya que implica desde la siembra y los cuidados para el crecimiento de los árboles de moreras, mínimo tres años, incluyendo también todo el proceso para el crecimiento de los gusanos de seda, alimentación y limpieza; la organización familiar ha permitido una buena coordinación ya que es necesario alimentarlos tres veces al día durante varias semanas. Organizan sus tiempos, mientras unos cortan y lavan hojas de mora, otros dan de comer a los gusanos, como se puede ver en el diagrama 3 


\section{Diagrama 3}

\section{Elaboración de artesanías de seda}

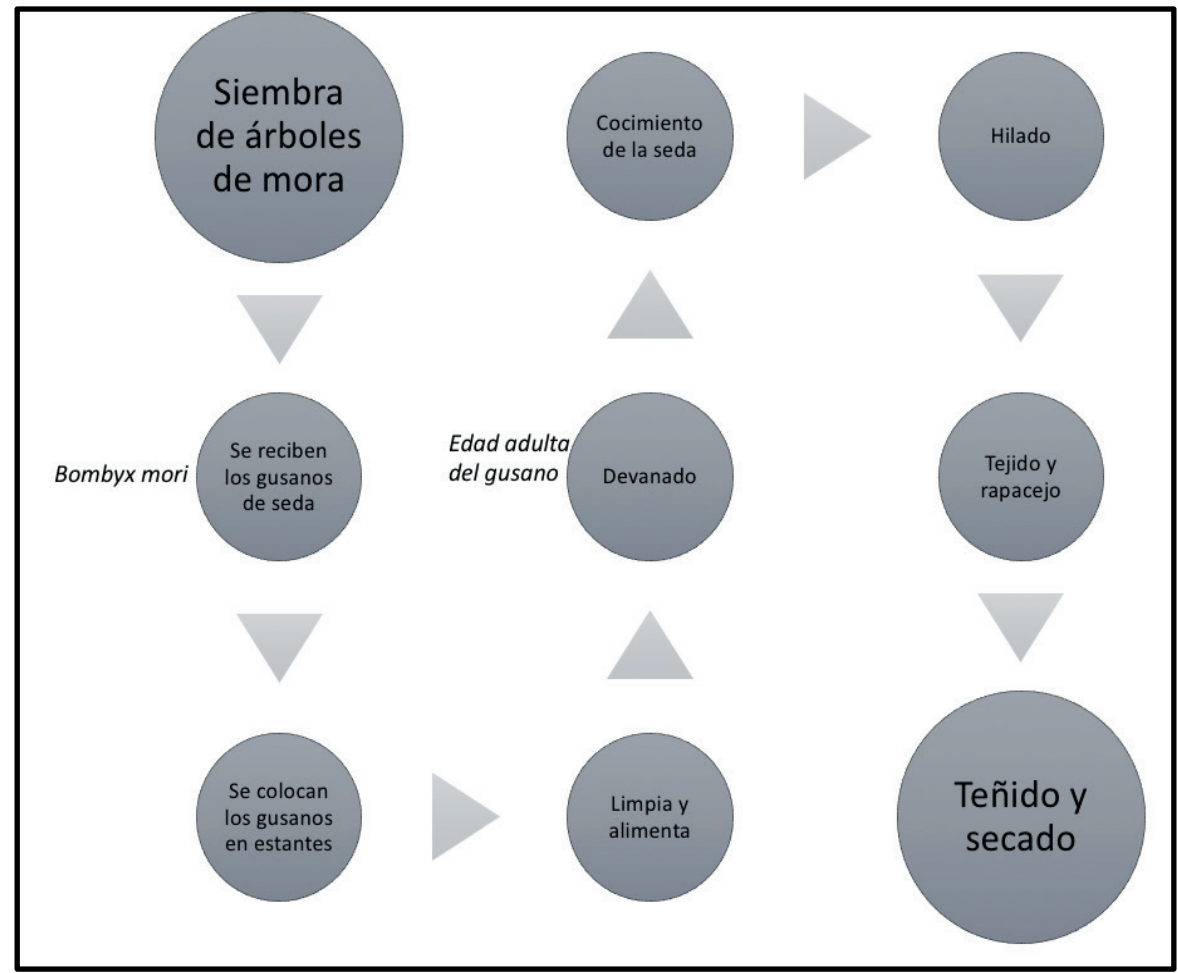

Fuente: elaboración propia basada en la Información observada en investigación de campo 2019.

El proceso para obtener la seda y así continuar con el hilado, se utilizan telares de cintura, y para mejorar la producción existen modelos mejorados como son los telares de pedal, además de requerir ruecas eléctricas y muchos otros artesanos siguen utilizando el malacate técnica ancestral.

Un aspecto que se observó durante las visitas de campo en su contexto cultural es la lengua materna, la forma de interactuar de los habitantes y de relacionarse, es por medio de esta, y más importante es que conservan aún su lengua materna (zapoteco). En particular el aprendizaje se transmite de generación en generación donde un maestro artesano enseña sus técnicas y saberes de la artesanía.

Durante las visitas de campo se identificaron a las unidades de análisis que según Babbie (2010:48) son lo que se examina para crear descripciones sumarias de ellas y para explicar sus diferencias. Para esta investigación la unidad de análisis son las redes familiares de artesanos sin importar si tienen una estructura formal o legal 
como organización o empresa, pero sí que tengan ciertos criterios de inclusión; trabajar bajo los principios de economía solidaria (igualdad, empleo, medio ambiente, cooperación, etc.) y con un enfoque comunitario, ya que, en la comunidad hay recursos naturales que se comparten en redes familiares $y$ comunitarias en un sistema productivo formado por redes familiares de artesanos.

Se tienen identificadas redes familiares y el número de integrantes, lo cuales se identifican con un nombre en específico como se muestra a continuación, (tabla 1):

\section{Tabla 1 \\ Redes familiares de artesanos en San Pedro Cajonos, Villa Alta, Oaxaca}

\begin{tabular}{|c|c|c|c|}
\hline Nombre & $\begin{array}{l}\text { Número de inte- } \\
\text { grantes }\end{array}$ & $\begin{array}{l}\text { Representante de la red } \\
\text { familiar }\end{array}$ & Actividad \\
\hline Wen Do Sed & 20 & Inés García & Artesanías de seda \\
\hline Aranda & 19 & Jaqueline Hernández Cruz & Artesanías de seda \\
\hline Flor de la morera & 12 & $\begin{array}{l}\text { Genoveva Martínez Her- } \\
\text { nández }\end{array}$ & Artesanías de seda \\
\hline Tone Toze & 7 & Gisela Rodríguez Martínez & Artesanías de seda \\
\hline Cuarta Generación & 6 & $\begin{array}{c}\text { María Del Carmen Martí- } \\
\text { nez Robles }\end{array}$ & Artesanías de seda \\
\hline Alborada & 7 & $\begin{array}{l}\text { Yulma Elizabeth Ruiz } \\
\text { Martínez }\end{array}$ & Artesanías de seda \\
\hline El rebozo arte y encanto & 9 & Inés García & Artesanías de seda \\
\hline Capullo de Seda & 9 & Jaqueline Hernández Cruz & Artesanías de seda \\
\hline Rebozo de Rienda & 3 & $\begin{array}{l}\text { Genoveva Martínez Her- } \\
\text { nández }\end{array}$ & Artesanías de seda \\
\hline $\begin{array}{l}\text { Artesanos en el tallado de } \\
\text { madera "Familia Blas" }\end{array}$ & 22 & Gisela Rodríguez Martínez & Artesanías de madera \\
\hline Total & 114 & & \\
\hline
\end{tabular}

Fuente: elaboración propia basada en la Información observada en investigación de campo en San Pedro Cajonos, (2019).

Ya que se conoce el total de la población (114) se consideran únicamente artesanos que realicen la actividad artesanal bajo el contexto de economía solidaria y con un enfoque comunitario, por lo que se trabajo, con la observación y para la investigación se utilizó una investigación cualitativa y descriptiva, se hicieron entrevistas a 10 artesanos representantes de cada red familiar, también se hizo observación participante para entender el proceso de producción y forma de organizarse para el trabajo de los textiles de hilo de seda y figuras de madera.

Como se puede observar se tienen identificadas 10 redes familiares, cada uno con diferente número de integrantes, 
se identificó a un representante por red para obtener información y llegar a una contrastación entre la teoría y la realidad de la vida social de las 10 redes familiares.

\section{Redes familiares y economía social: resultados}

Como resultado de un recorrido teórico de la economía solidaria y junto con el trabajo de campo realizado para esta investigación se tiene la contrastación de la vida social de las redes familiares de artesanos y su contexto de economía solidaria.

Como menciona Orellana (2007:4) la economía solidaria como una forma viable de organización económica, alternativa al sistema capitalista global y reflexionar sobre sus oportunidades de supervivencia, rescatando el protagonismo de la sociedad civil como motor de cualquier cambio y el rol de una cooperación verdaderamente transformadora que trabaje desde dos ámbitos en conjunto con la sociedad civil: por un lado, atacando los mecanismos que permiten la reproducción del sistema a escala planetaria (presionando para el cambio político, que juega un rol fundamental en la transformación) y, por otro, apoyando alternativas para configurar un cambio económico, diagrama 4.

\section{Diagrama 4 \\ EI Factor C de la economía solidaria}

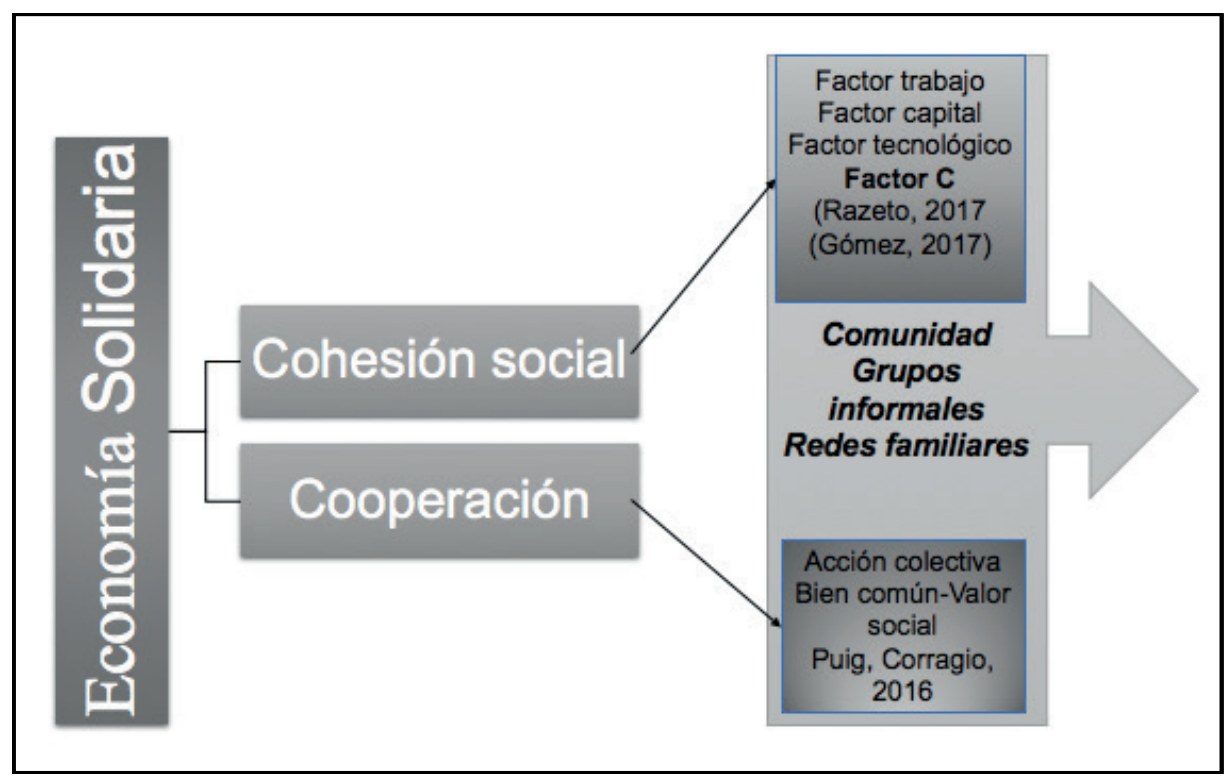

Fuente: Razeto (2017), Gómez (2017), Puig et al. (2016), Corragio (2016) 
Así, es como la economía solidaria es concebida en una idea de comunidad donde la cooperación y luego especificado en la variable de cohesión social (Factor C), junto con el factor trabajo, factor capital y factor tecnológico (Gómez, 2017:4) como se muestra en el diagrama 4.

Y, como lo menciona Hillenkamp (2012) en Puig et al. (2016:69) es indispensable para salir de una neomodernización, dinámicas de cooperación y cohesión social, y que se dan por el tipo de contexto institucional en interior de las comunidades, estas tienen las características propicias para que un contexto de comunidad solidaria de genere.

La conceptualización de esta forma del proceso de trabajo, de unificación activa de las condiciones de producción material (artesanías), en acto del trabajo, si bien hace referencia a un periodo histórico y a una comprensión teórica de la realidad íntima de la obtención de los medios de vida de muchos pueblos del mundo, no anula, en lo más mínimo, la existencia de amplias y hasta decisivas diferencias culturales, políticas, organizativas, religiosas o tecnológicas que existen entre estas sociedades que contienen el mismo núcleo productivo inmediato, pero que se reproducen en un entorno económico global que toma cuerpo social de una manera tremendamente distinta y variada entre unas y otras, conformando lo que Marx denomina tipos particulares de la forma general (García, 2009:243).

Tal como se ha señalado, la práctica es esencialmente productiva: produce realidad social. En esta producción de lo social. En especifico se habla especialmente, de la constitución que constituye una construcción constructiva (Potter, 1998:85). Es decir, el habla es construida por los sujetos según determinadas reglas principalmente sociales para que tenga mayor eficacia en la construcción de situaciones sociales.

Según esta concepción, hay discursos mejor construidos que otros $\mathrm{y}$, por tanto, más eficaces que otros en la construcción de situaciones sociales. En otras palabras, el discurso (el habla) no es sólo 'acerca de algo', sino que también 'hace algo' en el mundo: interviene de manera práctica (Potter, 1998:90), pero es más, el discurso no solamente construye hechos $y$ situaciones, sino que también pasa a formar parte constitutiva de estos hechos y situaciones: es una referencia a algo $\mathrm{y}$, al mismo tiempo, forma parte de ese algo (Potter, 1998:92). Esta concepción corresponde a un discurso social de construir situaciones sociales en un contexto comunitario.

En lo que sigue, las comunidades de práctica se caracterizaron a partir del trabajo realizado por Wenger, 1998) citado en Martínez (2006: 109). Según este autor, la persona que trabaja en organizaciones grandes, aprende sobre cómo desarrollar su práctica en comunidades más específicas, conformadas por personas que interactúan regularmente en el seno de estas organizaciones. Estas comunidades de práctica son, más bien, de tipo informal y distintas de las unidades organizacionales de los programas o instituciones (Martínez, 2006).

Por ello, desde un enfoque comunitario, se da pauta a un modelo de acción que hace referencia a los conocimientos y aprendizajes producidos en la práctica y que, a su vez, contribuyen a producirla. La producción de conocimientos y aprendizajes tiene 
lugar en el contexto de las comunidades de práctica.

Las intervenciones comunitarias se insertan, a su vez, en esta matriz de producción social: producen, construyen (y deconstruyen) contextos, pero a su vez son configuradas por los contextos comunitarios (Martínez, 2006:20).

Desde este enfoque, las iniciativas de desarrollo local se pueden considerar como las respuestas de los actores públicos y privados a los problemas y desafíos que plantea la integración de los mercados en la actualidad (Aydalot, 1985:487). La cuestión no reside en un sistema productivo de una localidad o un territorio que esté formado o no esté formado por empresas grandes o pequeñas, sino por la organización del sistema productivo local.

Se puede decir que las unidades familiares/comunitarias y el emprendimiento económico entre diversas organizaciones de un mismo territorio o sector, resuelven condiciones generales con productores que comercializan $o$ se abastecen juntos y que se orienten al desarrollo de la comunidad local, según Coraggio (2013:9).

Así, los sistemas productivos locales, los distritos industriales son formas de organización de la producción, con base en la división del trabajo entre las empresas y la creación de un sistema de intercambios locales que impulsa el aumento de la productividad y el crecimiento de la economía. Se trata de un modelo de organización que permite generar rendimientos crecientes cuando la interacción entre las empresas propicia la utilización de las economías externas de escala existentes en los sistemas productivos que, a fin de cuentas, es uno de los potenciales de desarrollo de las economías.
Al proporcionarle, a la economía, el adjetivo de "solidaria" con el objetivo de determinar una economía que sea incluyente de valores y prácticas solidarias, con enfoque colectivo, se habla de un sistema económico presente el principio ético de reproducción y desarrollo de la vida que será, necesariamente, social y solidario (Coraggio, 2008:127).

Por todo lo dicho anteriormente, en esta investigación, la unidad de estudio se establece con base en un contexto social solidario, comunitario y popular y, por tanto, la unidad básica de organización económica popular será la unidad domestica (familia o comunidad) que en las comunidades son el pilar principal del desarrollo económico local.

\section{Conclusión}

En San Pedro Cajonos, se observó una fuerte dinámica de cooperación y cohesión social, que por el tipo de contexto poseen características fundamentales para que se genere en su interior un contexto de economía solidaria.

En la comunidad, en especial en las redes familiares de artesanos, a partir de la formación de grupos informales y mediante la acción colectiva, se tiene presente el bien común como aspecto central que genera valor social a actividades productivas, generando el bien común. Esta toma de posición demuestra que se genera un proceso de integración donde emerge el sentido de pertenencia.

Lo anterior se manifiesta de forma comunitaria de producción y propiedad ancestral de ahí que resulte una forma de vida colectiva en red con dos procesos de producción definidos con gran la riqueza cultural, políticos, 
simbólicas y organizativas en el seno de la comunidad.

El diseño e implementación de las estrategias comunitarias, deben tomar en cuenta estas condiciones de reflexividad que se despliegan por el hecho de intervenir en el contexto de los sistemas naturales con los que se trabaja, sistemas que constituyen el ambiente en el que viven las comunidades desde el aprovechamiento de sus recursos hasta las actividades productivas que tengan o que puedan realizarse a partir de dichos recursos que les proporciona el sistema, como es el caso de los artesanos al momento de la elaboración de artesanías, ya que en la comunidad encuentran las principales materias primas, desde el árbol de aguacatillo para el tallado de madera y el árbol de morera para alimentar a los gusanos de seda.

Los artesanos de madera por su parte de lo que observan en su vida cotidiana y en la inspiración de su vida social tallan la madera creando piezas únicas del entrono como libélulas, jaguares, mariposas, ranas, serpientes, por mencionar algunos. Y los artesanos de hilo de seda tiñen de forma natural sus artesanías textiles con solo hervir algunas hojas o cortezas de plantas que la misma naturaleza les proporciona.

Con esta investigación se está en la búsqueda de una posible vía alterna que no esté enmarcada en la economía de mercado que se caracteriza por el ánimo de lucro y enriquecimiento individual, sino, más bien, en la economía solidaría para promover, con su orientación al bien común, y colectivo y que agrega componentes con los cuales se genera cooperación y cohesión social y esté encaminada al valor social en una comunidad.

\section{Referencias bibliográficas}

Aydalot, P. (1985). Economía regional y urbana. París: Económica.

Babbie, E. (2010) Fundamentos de investigación social. Thomsom Editores. http://online.aliat.edu. $\mathrm{mx/}$ adistancia/InvCuantitativa/lecturas S5/Babbie Fundamen de la invest social.pdf

Calderon, C. L. F. (2008). La economía solidaria: de lo legal a la formación integral. Criterio Libre, 6(9), 47-72. https://dialnet.unirioja.es/servlet/ articulo?codigo $=4547093$

Celorio, G., \& López de Munain, Alicia, A. (2007). Diccionario de educación para el desarrollo. Bilbao: Ed. Hegoa. https://publicaciones.hegoa.ehu.eus/ publications/172

Coraggio, J. (2008). Economía social, acción pública y política. Hay vida después del neoliberalismo. 2da. ed. Ediciones CICCUS. https:// www.socioeco.org/bdf fichepublication-455 es.html

Coraggio, J. (2013b). La economía social y solidaria y el papel de la economía popular en estructura económica. Quito: IEPS. https:// www.coraggioeconomia.org/jic/ archivos \%20para\%20descargar/ La\%20economia\%20Popular\%20 y\%20Solidaria $\% 20$ El $\% 20$ Ser $\% 20$ Humano $\% 20$ Sobre $\% 20$ el $\% 20$ Capital.pdf

Fajardo, C. (2008). La economía solidaria: de lo legal a la formación integral. Criterio Libre, (9). http:// www.socioeco.org/bdf fichedocument-3382 es.html

García, A. (2009). Forma valor y forma comunidad. La Paz, Bolivia: Muela del Diablo Editores. http://biblioteca. clacso.edu.ar/gsdl/collect/clacso/ index/assoc/D1242.dir/garcial2.pdf 
Gómez, N. (2017). Luis Razeto Migliaro, Tópicos de Economía Comprensiva. Ediciones Universitas Nueva Civilización, 2015, Santiago, 208 p. Polis, 46. http://journals.openedition. org/polis/12412

Hillenkamp, I., \& Bessis, F. (2012). L'innovation sociale par l'economie solidaire en Bolivie. Innovations, 2(38), 85-105. https://www.cairn.info/ journal-innovations-2012-2-page-85. $\underline{\mathrm{htm}}$

Instituto Nacional de Estadística y Geografía, INEGI. (2015). XII Censo de Población y Vivienda 2015. https:// www.snim.rami.gob.mx

Klein, J., Laville, J., \& Moulaert, F. (2014). L'innovation sociale: repères introductifs. Toulouse: Erès. https://www.cairn.info/l-innovationsociale--9782749239491.htm

Martínez, V. R. (2006). El enfoque Comunitario, El desafío de Incorporar a la Comunidad en las Intervenciones Sociales. Chile: Facultad de Ciencias Sociales. http://dspace.utalca.cl/ bitstream/1950/9417/1/martinez ravanal.pdf

Orellana, M. (2007). La economía solidaria como forma de organización económica alternativa al sistema capitalista global. Universitat de Barcelona, Master Globalización.
Desarrollo y Cooperación Observatori Solidaritat UB. http://www.socioeco. org/bdf fiche-document-906 es.html

Potter, J. (1998). La representación de la realidad. Discurso, retórica y construcción social. Paidós. https://dialnet.unirioja.es/servlet/ libro?codigo $=64242$

Puig, C. (Coord.)., Coraggio, J. L., Laville, J.-L., Hillenkamp, I., Farah, I., Jimenez, J., Vega, S., Guridi, L., y Perez-Mendiguren, J. C. (2016). Economía Social y Solidaria: Conceptos, prácticas y políticas públicas. España: Hegoa, Universidad del País Vasco. https:// publicaciones.hegoa.ehu.eus/es/ publications/351

Razeto, L. (1989). La Economía de la Solidaridad. IV Congresi de Economistas Javerianos. Bogotá. https://www.javeriana. edu.co/Facultades/fear/ ier/?idcategoria $=13410$

Yaselca, P., \& Jara, I. (2013). El proceso de los circuitos económicos solidarios interculturales. Univesidad Politécnica Salesiana, Quito. https://dspace.ups.edu.ec/ bitstream/123456789/10826/1/ El\%20proceso $\% 20$ de $\% 201$ os $\% 20$ circuitos $\% 20$ economicos $\% 20$ solidarios\%20interculturales.pdf 